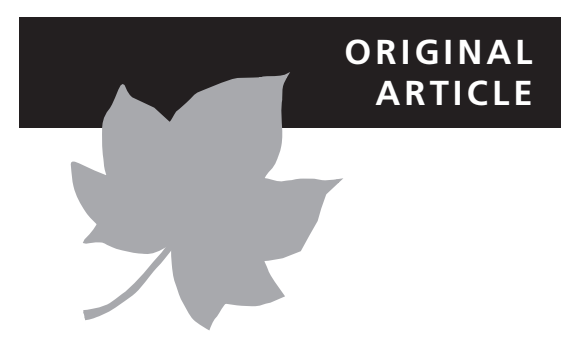

\title{
Phylogenetic structure of vertebrate communities across the Australian arid zone
}

\author{
Hayley C. Lanier*, Danielle L. Edwards and L. Lacey Knowles
}

Department of Ecology and Evolutionary Biology, Museum of Zoology, University of Michigan, Ann Arbor, MI 48109-1079, USA

\begin{abstract}
Aim To understand the relative importance of ecological and historical factors in structuring terrestrial vertebrate assemblages across the Australian arid zone, and to contrast patterns of community phylogenetic structure at a continental scale.
\end{abstract}

Location Australia.

Methods We present evidence from six lineages of terrestrial vertebrates (five lizard clades and one clade of marsupial mice) that have diversified in arid and semi-arid Australia across 37 biogeographical regions. Measures of within-lineage community phylogenetic structure and species turnover were computed to examine how patterns differ across the continent and between taxonomic groups. These results were examined in relation to climatic and historical factors, which are thought to play a role in community phylogenetic structure. Analyses using a novel sliding-window approach confirm the generality of processes structuring the assemblages of the Australian arid zone at different spatial scales.

Results Phylogenetic structure differed greatly across taxonomic groups. Although these lineages have radiated within the same biome - the Australian arid zone - they exhibit markedly different community structure at the regional and local levels. Neither current climatic factors nor historical habitat stability resulted in a uniform response across communities. Rather, historical and biogeographical aspects of community composition (i.e. local lineage persistence and diversification histories) appeared to be more important in explaining the variation in phylogenetic structure. While arid-zone assemblages show an overall tendency towards phylogenetic clustering, this pattern was less pronounced at finer spatial scales.

Main conclusions By focusing within different taxonomic groups and between those groups within regions, we show that although the vertebrate lineages we examined exhibited high diversity and low turnover across the arid zone, the underlying phylogenetic structure differs between regions and taxonomic groups, suggesting that taxon-specific histories are more important than habitat stability in determining patterns of phylogenetic community relatedness.

\section{Keywords}

Arid zone, Australia, community phylogenetic structure, Ctenotus, Ctenophorus, Diplodactylinae, IBRA regions, Lerista, Pygopodidae, Sminthopsinae.

\section{INTRODUCTION}

The composition of a regional biota is the result of ecological and biogeographical processes - that is, it reflects proximate

(c) 2013 Blackwell Publishing Ltd species tolerances and competitive interactions as well as the influence of regional and historical events. Because these influences are intrinsically linked (environmental tolerances mediate the distributions of organisms; regional species 
occurrence facilitates local species presence; Wiens, 2011), analyses seeking to infer processes contributing to community structuring need to include information at multiple scales and from multiple approaches. Recent approaches contrasting the relative roles of abiotic and biotic influences with neutral patterns in structuring communities have gained considerable traction in ecological literature (Webb et al., 2002). By integrating information on phylogenetic ancestry with species co-occurrence, community phylogenetic analyses have revealed patterns of community structure across the biotic and abiotic gradients (Bryant et al., 2008; Graham et al., 2009) resulting from contemporary ecological processes and biogeographical histories (Webb et al., 2008). Inference from these local-scale patterns may be informative on a regional or continental scale (Vamosi et al., 2009; Cardillo, 2011), but the degree to which these patterns are indicative of regional or continental processes has been little studied (Vamosi et al., 2009). Furthermore, their generality across multiple co-occurring taxonomic groups in a given ecosystem is unknown.

Community phylogenetic analyses have revealed a wide range of variables that may be important for structuring species-rich communities (e.g. habitat complexity, moisture availability, nutrient richness and competition between species; Webb et al., 2008; Graham et al., 2009; Kooyman et al., 2011). The Australian arid zone (Fig. 1) presents a perplexing contrast to these patterns, with habitats characterized by exceedingly infertile soils, low moisture availability and highly variable rainfall, supporting some of the most diverse lizard communities in the world (Morton et al., 2011). Furthermore, the relatively inhospitable climates of the Holocene are thought to be moderate in comparison to the extreme aridity that typified the region in the Pleistocene (reviewed in Byrne, 2008). Arid-zone communities are formed from

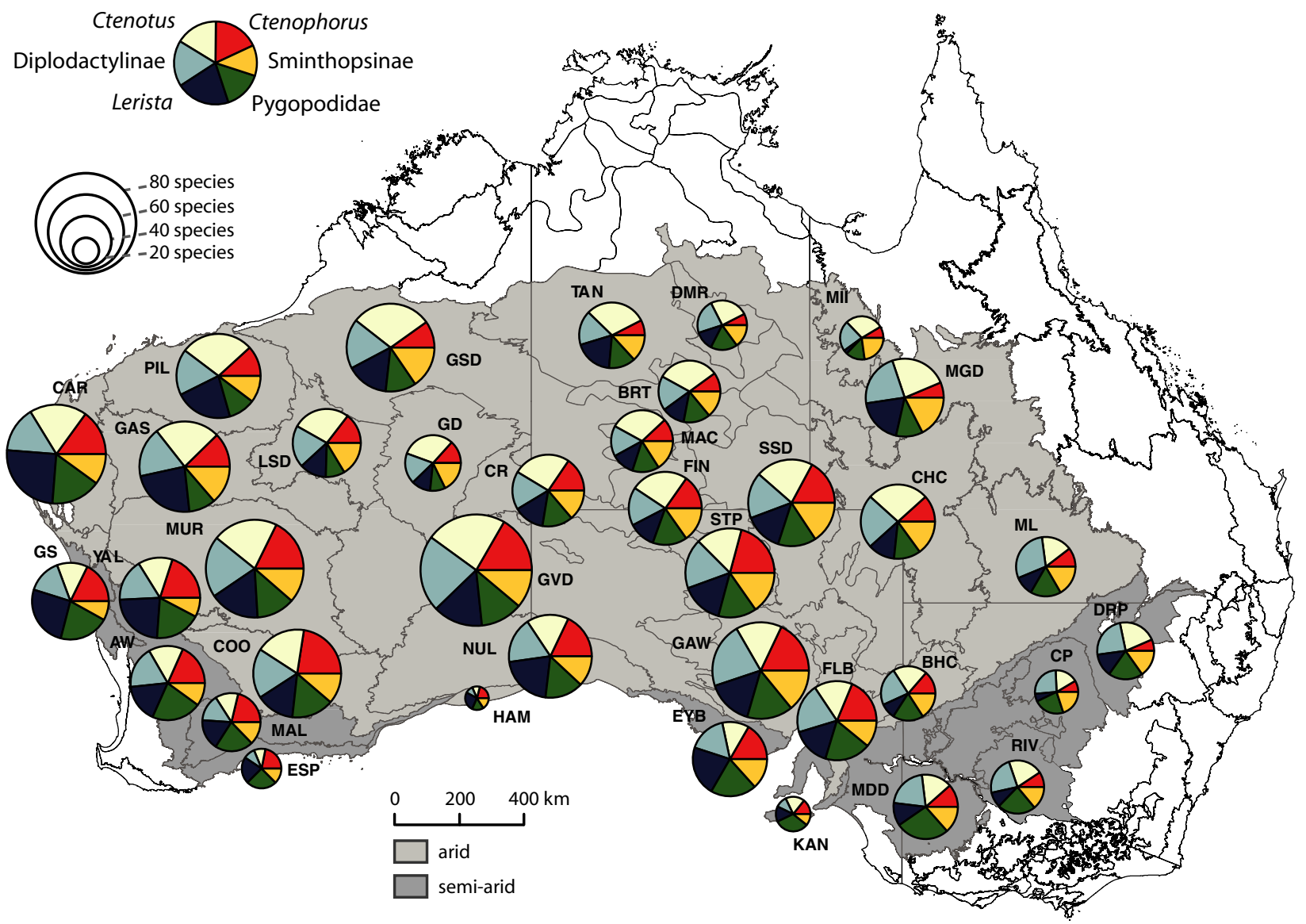

Figure 1 Map showing the correlations of alpha diversity $(\rho=0.51)$ in the six focal taxonomic groups across the Australian arid zone (grey). Species diversity is shown as a proportion of total regional diversity (pie size) by IBRA (Interim Biodiversity Regional Assessment) region. AW, Avon Wheatbelt; BHC, Broken Hill Complex; BRT, Burt Plain; CAR, Carnarvon; CHC, Channel County; COO, Coolgardie; CP, Cobar Peneplain; CR, Central Ranges; DMR, Davenport Murchison Ranges; DRP, Darting Riverine Plains; ESP, Esperance Plains; EYB, Eyre Yorke Block; FIN, Finke; FLB, Flinders Lofty Block; GAS, Gascoyne; GAW, Gawler; GD, Gibson Desert; GS, Geraldton Sandplains; GSD, Great Sandy Desert; GVD, Great Victoria Desert; HAM, Hampton; KAN, Kanmantoo; LSD, Little Sandy Desert; MAC, MacDonnell Ranges; MAL, Mallee; MDD, Murray Darling Depression; MGD, Mitchell Grass Downs; MII, Mount Isa Inlier; ML, Mulga Lands; MUR, Murchison; NUL, Nullarbor; PIL, Pilbara; RIV, Riverina; SSD, Simpson Strzelecki Dunefields; STP, Stony Plains; TAN, Tanami; YAL, Yalgoo. 
taxa that have adapted to aridity from largely mesic ancestors (Byrne et al., 2008) and diversified despite fluctuating productivity and resource uncertainty, and without the presence of any obvious barriers to dispersal such as mountain ranges or glaciers (Pianka, 1972).

Instead of being filtered by large climatic gradients (temperature, elevation and precipitation are relatively homogeneous across the region; Morton et al., 2011), differing sediment types (sand plains, sand ridges and/or stony deserts) and their associated vegetation (spinifex grassland and shrublands) may have acted to structure terrestrial aridzone communities (Shoo et al., 2008). These habitats also differ in terms of origin, with highly mobile sandy deserts emerging recently (based on climatic and geological evidence; Macphail, 1997, and Fujioka et al., 2009, respectively), whereas isolated regions of greater topological complexity appear to have provided relative climatic stability over the extreme aridification cycling of the last $15 \mathrm{Myr}$ (Pepper et al., 2011). Because of these ecological characteristics, the Australian arid zone has become a classic study system for assessing the role of ecological barriers (Pianka, 1969, 1972), competition (Rabosky et al., 2011) and habitat specialization (Dickman et al., 2001; Rabosky et al., 2007) in the development of community structure. Furthermore, it is these ecological and historical characteristics that make this study system especially amenable to continental-scale investigations of community phylogenetic structure.

Here, we use a community phylogenetic approach (Webb et al., 2002; Vamosi et al., 2009; Kembel et al., 2010) to examine the degree to which shared biogeographical forces and environmental factors result in similar broad-scale community structure across the Australian arid zone. Community phylogenetics brings together theories from ecology and evolution to examine the phylogenetic patterns underlying community structure (Webb et al., 2002; Vamosi et al., 2009). By comparing the phylogenetic relatedness of a community to expectations of assembly under neutral processes from a regional pool, communities can be characterized as either more or less phylogenetically structured than expected by chance. When examined on a local level, these patterns are often interpreted as evidence of either habitat filtering for similar ecological tolerances (phylogenetic clustering) or competitive exclusion of ecologically similar close relatives (phylogenetic overdispersion). At the regional or continental scale, these phylogenetic patterns have been thought to provide evidence of intra-continental diversification or extensive allopatric speciation, respectively (Webb et al., 2008; Cardillo, 2011), although this inference is limited by the small number of studies that have been conducted at this analytical scale (Vamosi et al., 2009). Furthermore, different patterns of community structure may dominate at different scales, with a tendency towards phylogenetic clustering at the broadest scales (Vamosi et al., 2009). Regional histories are also important, with greater phylogenetic evenness in regions of habitat stability (e.g. refugia; Kooyman et al., 2011). Ultimately, all of these patterns are likely to be affected by the biological traits of a species group (e.g. environmental tolerances and dispersal capability; Wiens, 2011), their evolutionary lability (e.g. niche conservatism; Wiens \& Graham, 2005), and the strength of interactions with other species.

We extend the traditional community phylogenetic framework to the dominant vertebrate constituents across the Australian arid zone (Table 1), as opposed to the typical approach of a detailed analysis on a single large phylogeny conducted at a local level, in order to assess similarity across multiple groups. The targeted taxonomic groups are species rich (a necessity for powerful tests of community assembly), small-bodied, terrestrial and heterothermic, with correlated patterns of species diversity in the arid zone (Fig. 1; Pearson's correlation coefficient $\rho=0.53$ ). Four of the groups radiated following the onset of aridity, whereas two (diplodatyline geckos and the pygopodid legless lizards) are thought to have adapted to aridification in situ (Byrne et al., 2008). Convergent evolution between taxonomic groups is also apparent. For example, two of the groups - Lerista and the Pygopodidae - have experienced limb reduction or loss (Jennings et al., 2003; Skinner \& Lee, 2009). Considering assemblages drawn from six taxonomic groups that inhabit a similar trophic level across the arid zone provides a context to ask whether community patterns from one taxonomic group can conceivably be generalized to another similar

Table 1 Focal taxa from the Australian arid zone, showing the number of species included, the basal age of the lineage, and taxonomic descriptions.

\begin{tabular}{|c|c|c|c|}
\hline & $\begin{array}{l}\text { No. of } \\
\text { taxa }\end{array}$ & $\begin{array}{l}\text { Basal age } \\
(\mathrm{Ma})\end{array}$ & Evolutionary ecology \\
\hline Diplodactylinae & 59 & $16-27$ & Diplodactyline geckos diverse in the arid zone but with Gondwanan evolutionary origins \\
\hline Pygopodidae & 33 & $10-23$ & Gondwanan-origin legless gekkonids with a broad range of body size variation \\
\hline Lerista & 77 & 20 & $\begin{array}{l}\text { Recently radiated genus of sphenomorphine skinks with broad morphological variability and } \\
\text { fossorial to subfossorial life histories }\end{array}$ \\
\hline Ctenotus & 44 & 20 & $\begin{array}{l}\text { Recent hyperdiverse genus of sphenomorphine skinks with conserved body forms and broad } \\
\text { distributions within the arid zone }\end{array}$ \\
\hline Ctenophorus & 35 & $11-12$ & Recent adaptively radiated genus of agamid lizards occupying a range of niches within the arid zone \\
\hline Sminthopsinae & 23 & $6-10$ & Recently radiated insectivorous marsupial mice from the dasyurid family \\
\hline
\end{tabular}


group across the same landscape. We calculate these metrics for each arid and semi-arid IBRA (Interim Biodiversity Regional Assessment) region, delimited based upon geological, biotic and climatic factors; these regions allow comparisons between discrete, biologically meaningful segments of the arid zone. Because patterns of community phylogenetic structure and ecological processes are known to be scalespecific (Cavender-Bares et al., 2006), we couple this approach with a sliding-window technique to examine phylogenetic community structure at a variety of scales ranging from the regional (the IBRA region size) to a local level $\left(50 \mathrm{~km}^{2}\right)$. This context provides a contrast between local and regional phenomena and avoids confounding biological phenomena with statistical artefacts (i.e. misleading results from inappropriate geographical scales of analysis; see Swenson et al., 2006; McGill, 2010). By contrasting the phylogenetic structure in six vertebrate groups at varying scales, we intend to test: (1) whether phylogenetic structure is similar across scales, (2) whether similar taxa exhibit similar community phylogenetic structure, and (3) which historical and/or ecological factors best explain the observed patterns.

\section{MATERIALS AND METHODS}

\section{Identifying species distributions and taxonomic assemblages}

Raster maps of species distributions (data deposited in the Dryad Repository: http://dx.doi.org/dryad.35321) were used to score the presence or absence of species in smallvertebrate communities across the 5.4 million $\mathrm{km}^{2}$ of the Australian arid zone. Marsupial occurrences, based on the polygon data sets distributed by the IUCN Red List (http:// www.iucnredlist.org/), and rasterized polygons created from reptile distribution records based on OZCAM data (http:// www.ozcam.org.au/) were vetted against published distributions (Menkhorst \& Knight, 2004; Wilson \& Swan, 2008). Recent taxonomic revisions were incorporated for Lerista (Smith \& Adams, 2007), Ctenophorus (D.L. Edwards, unpublished data), Diplodactylus (Doughty et al., 2008; Hutchinson et al., 2009) and Lucasium (Doughty \& Hutchinson, 2008). Community phylogenetic scores were calculated for assemblages of each taxonomic group across the 37 arid and semiarid IBRA regions (v. 6.1; http://www.environment.gov.au/ metadataexplorer/explorer.jsp) (Fig. 1). These regions are based upon the climatic, geological, landform, floral and faunal makeup of the landscape, grouped to form 85 distinct bioregions that are used for conservation status designations, land use planning and natural resource management.

\section{Phylogenetic trees}

Phylogenies were estimated for each of the six taxonomic groups (Table 1; http://dx.doi.org/dryad.35321) from data available on GenBank. Re-estimating phylogenies, as opposed to using previously published trees, provides a standardiza- tion of estimated branch lengths in each of the six clades (making phylogenies comparable for computing community metrics) and expands taxonomic coverage by including sequence data from multiple publications. Sequences were aligned using the KALIGN algorithm implemented in EBIoX and checked by eye; nucleotides lacking unambiguous alignment were excluded. Models of nucleotide substitution for each locus were determined using the Akaike information criterion in the program MrModeltest 2.2 (Nylander, 2004). Phylogenetic analyses in BeAst (Drummond \& Rambaut, 2007) were run using a Yule model and a lognormal relaxed clock, partitioned by gene (see Appendix S1 in Supporting Information) separately for each taxonomic group, for a minimum of 10 million generations, sampling every 1000 and with a $10 \%$ burn-in excluded. The resulting trees were vetted against the original publications to ensure that highly supported nodes were represented (for a discussion of phylogenetic resolution and analytical power, see Swenson, 2009).

\section{Computation of community phylogenetic metrics}

We use two community phylogenetic measures to quantify whether assemblages are either more or less related than would be expected by chance - the net relatedness index (NRI, sensitive to phylogeny-wide patterns; Webb et al., 2002) and the nearest taxon index (NTI, sensitive to structure at the tips of a phylogeny; Webb et al., 2002). Metrics and significance were assessed using the $\mathrm{R}$ package PICANTE 0.7-2 (Kembel et al., 2010) by comparing observed phylogenetic relatedness to a null distribution calculated by randomizing co-occurrence 1000 times using the independent swap algorithm while maintaining the overall frequency of species occurrence in the data set. This null model has been shown to have high power to detect niche-based community assembly and does not suffer from the high Type I error rates associated with many other community phylogenetic null models (Kembel, 2009). Because the null model can greatly affect the strength and power of community phylogenetic metrics, we compared these results to those calculated using an alternative null model where communities are composed of random draws from the species pool (Appendix S2). Significantly negative NRI or NTI values indicate a greater phylogenetic dissimilarity between community members than would be expected by chance (phylogenetic evenness or overdispersion). Significantly positive values indicate that closely related taxa occur together at a greater frequency than would be expected by chance (phylogenetic underdispersion or clustering; Webb et al., 2002). The correlation of regional NRI/NTI scores between taxonomic groups was examined with a randomization test in $\mathrm{R}$. The effect of species turnover over time and space was also examined by calculating community beta diversity (CBD) for each taxonomic group and contrasting those values with phylogenetic beta diversity (PBD) (Bryant et al., 2008; Graham \& Fine, 2008). 
Phylogenetic structure of the Australian arid zone

\section{Sliding window analyses}

A 'sliding window' spatial analysis of species presence/ absence was used to test the robustness of the community phylogenetic results to the effects of spatial scale across the Australian landscape. Referenced sampling grids at 50, 100, 250, 500 and $1000-\mathrm{km}$ scales were created for the continent using Hawth's Analysis Tools in ArcGIS 9.3 (ESRI, Redlands, CA, USA). Each grid location was set for an initial sampling, and with two replicate resampling grids created by shifting to the east or north by half the grid length (e.g. the $50-\mathrm{km}$ grid was shifted $25 \mathrm{~km}$ ) to create three overlapping reference grid data sets (http://dx.doi.org/dryad.35321). Assemblage NRI and NTI scores were calculated as described above with the total number of communities in the matrix held constant at 50 (data sets were randomly subdivided), to separate the effect of community size from the number of communities being compared.

\section{Post-hoc evaluation of factors contributing to community structure}

To investigate the role of climatic and historical factors in community phylogenetic structure, representative climatic, soil and elevation data were extracted for each of the 37 arid and semi-arid IBRA regions. The centroid of each regional polygon was used to provide a representative sample. Current climatic data on maximum temperature, minimum temperature, temperature seasonality, precipitation and precipitation seasonality were extracted from the WorldClim data set (Hijmans et al., 2005). The importance of climate in structuring arid-zone communities was assessed in two ways: (1) the relative importance of climatic variables in predicting NRI and NTI scores was examined using a regression-tree approach; and (2) the role of climatic similarity in driving phylogenetic similarity was examined through a series of partial Mantel tests on PBD. Regression trees were estimated in the RANDOMForest package for $\mathrm{R}$ (Cutler et al., 2007) to provide a robust means of evaluating the relationship between a response variable and a set of potentially informative predictor variables. RANDOMFOREST deals well with nonlinear relationships and predictor variables with non-normal underlying distributions (Cutler et al., 2007). A series of regression trees $($ ntree $=1000)$ from bootstrapped samples were used to examine the relationship between NRI or NTI and a combination of up to six explanatory variables that were chosen to maximize the gain of variance explained relative to the maximum explained variance using a full model. The relationship between regional climate, PBD, elevation and the centre of origin of a group was examined using a series of partial Mantel tests with 1000 permutations (following Bryant et al., 2008) to account for community composition (CBD) and spatial autocorrelation. The centre of origin of each taxonomic group was calculated as the geographical region (at the 50-km sampling scale) with the largest number of co-occurring taxa.

\section{RESULTS}

\section{Regional community structure}

Despite overall similarities in the geographical distribution of species diversity between taxonomic groups (Fig. 1), there is a general lack of concordance in the underlying community phylogenetic structure (in NRI or NTI) across taxonomic groups at the continental scale (Fig. 2). While some groups in certain regions show similar trends with regard to community structure (i.e. positive or negative NRI or NTI), these trends are not consistent across regions or across taxonomic groups. For example, although Sminthopsinae and Lerista exhibit similar levels of clustering along the west coast, an area where other taxonomic groups trend towards neutrality or overdispersion (Fig. 2), the overwhelming regional pattern across the arid zone as a whole is dissimilarity in sign of response and/or magnitude of phylogenetic structure between taxonomic groups. This general dissimilarity in phylogenetic structure is robust, irrespective of the specific metric (NRI or NTI) or null model (Appendix S2). Permutation tests confirm that community phylogenetic structure is rarely significantly positively correlated between taxonomic groups (Table 2). In general, community phylogenetic structure within each taxonomic assemblage based on NTI is similar to that calculated from NRI, with Pearson's correlation coefficient between the two indices ranging from a minimum of 0.36 for regional structure within Ctenotus to a maximum of 0.87 within Ctenophorus (Table 2).

\section{The geography of phylogenetic structure}

While taxonomic groups at the IBRA scale of analysis (i.e. regional scale) generally tend towards weakly clustered (i.e. positive) phylogenetic structure (NRI or NTI), all groups also show some overdispersion in some regions (Fig. 2, Appendix S3: Fig. S3). Similarly, although there are some commonalities in the geographical location of local phylogenetic overdispersion and clustering across taxonomic groups, there are also some notable differences among the taxa. For example, four of the taxonomic groups (Lerista, Diplodactylinae, Sminthopsinae and Pygopodidae) show overdispersion in the semi-arid south-east (Fig. 2), whereas assemblages of Ctenophorus lizards exhibit an inverse geographical trend, with neutral or overdispersed phylogenetic structure in the central arid zone and phylogenetic clustering in the south-east.

Regional habitat stability (i.e. putative thermal refugia versus unstable dune regions) did not result in a similar community phylogenetic structure across all taxonomic groups or regions (Fig. 3). When regions identified as centres of endemism or putative refugia are compared with nearby regions of recent habitat turnover (e.g. regions characterized by highly mobile sand dunes), no concerted change in phylogenetic structure is shared across taxonomic groups (as suggested by Kooyman et al., 2011). In general, the sand 

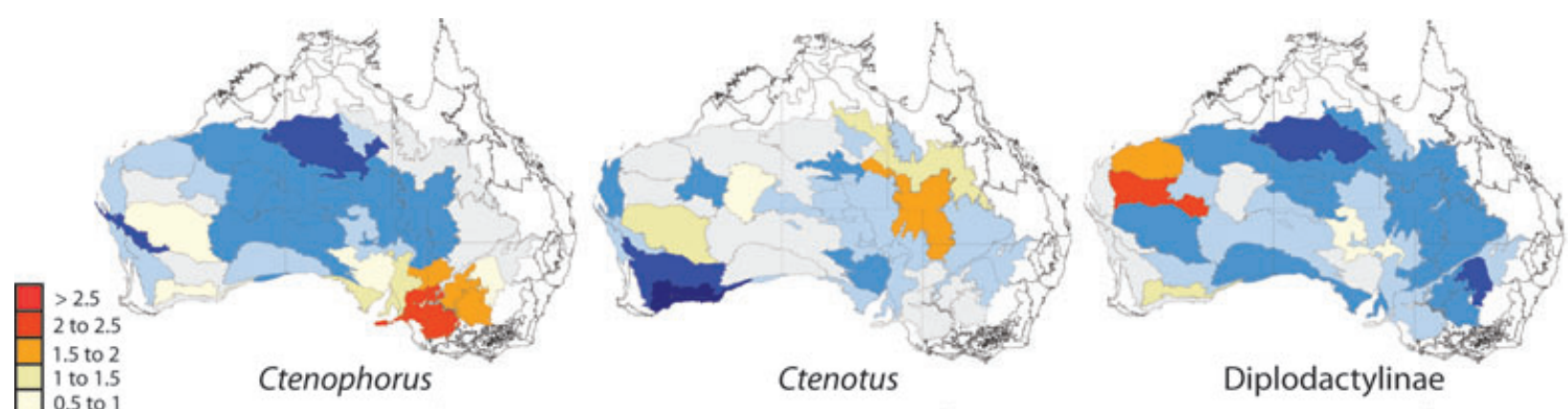

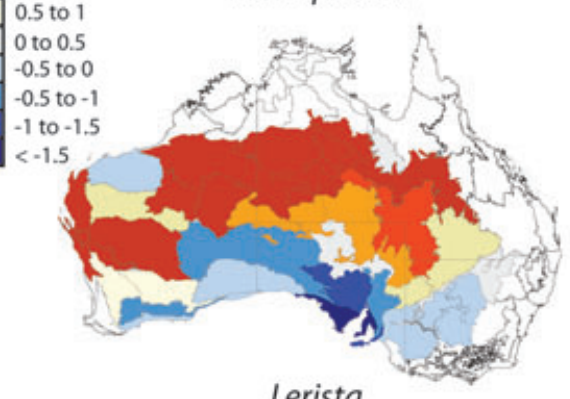

Lerista

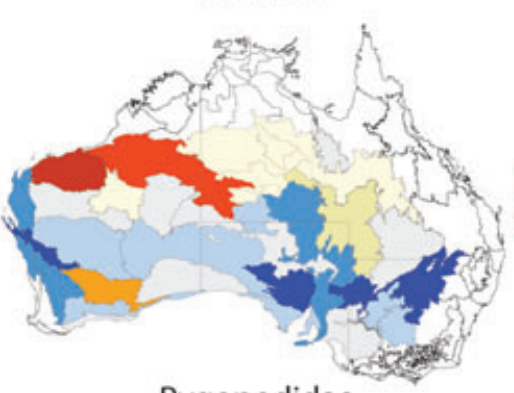

Pygopodidae

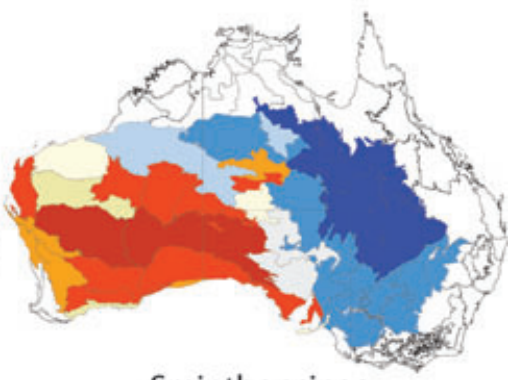

Sminthopsinae

Figure 2 Absolute values of the net relatedness index (NRI) ranging from overdispersed $(<1.5)$ to fairly neutral $(-0.5$ to 1.5$)$ to highly clustered (> 2.5) reveals phylogenetic structure across the Australian arid zone that is weakly correlated across the six focal taxonomic groups.

Table 2 Correlation between phylogenetic structure in the net relatedness index (NRI; above diagonal), nearest taxon index (NTI; below diagonal), and between NRI and NTI (on diagonal) within the six focal taxonomic groups across the IBRA regions of the Australian arid zone. Values significant at the $\alpha=0.05$ level (based on 1000 permutations) are shown in italics, with significantly positive correlations shown in bold.

\begin{tabular}{lrrrrrr}
\hline & Ctenop. & Ctenot. & Diplo. & Ler. & Pygo. & Smin. \\
\hline Ctenophorus & $\mathbf{0 . 8 7}$ & 0.07 & 0.04 & -0.58 & -0.24 & -0.26 \\
Ctenotus & -0.55 & $\mathbf{0 . 3 6}$ & -0.25 & 0.15 & 0.15 & -0.30 \\
Diplodactylinae & -0.20 & -0.22 & $\mathbf{0 . 7 5}$ & -0.22 & 0.03 & $\mathbf{0 . 3 2}$ \\
Lerista & -0.46 & 0.17 & 0.23 & $\mathbf{0 . 6 0}$ & $\mathbf{0 . 2 5}$ & 0.03 \\
Pygopodidae & -0.50 & $\mathbf{0 . 2 7}$ & -0.02 & 0.22 & $\mathbf{0 . 7 5}$ & -0.01 \\
Sminthopsinae & 0.14 & -0.34 & 0.11 & 0.14 & -0.28 & $\mathbf{0 . 6 2}$ \\
\hline
\end{tabular}

specialist Lerista tends to show phylogenetic clustering in the historically unstable dune regions, whereas the two most ancient groups - the Diplodactylinae and the Pygopodidae exhibit nearly identical phylogenetic responses (i.e. increasing or decreasing structure) across all comparisons (Fig. 3) irrespective of the stability of the habitat.

\section{Effects of scale}

Generally, community phylogenetic structure at the IBRA (i.e. regional) scale of analyses is similar to that from the sliding window analysis in terms of geographical patterns; the geographical locations for overdispersion/clustering in community phylogenetic metrics are similar in IBRA and region-free analyses (Fig. 4, Appendix S3: Figs S4-S9). For example, Sminthopsinae and Ctenophorus tend towards phylogenetic clustering and overdispersion, respectively, in the central arid zone, for both IBRA and sliding-window analyses. This qualitative similarity is supported by permutation tests, which indicated that IBRA regions exhibit phylogenetic structure that is significantly more similar to the region-free sampling than would be expected by chance alone for all six taxonomic groups.

As the size of the sliding window decreases (i.e. analyses are shifted to finer geographical scales), greater geographical resolution of locations of community phylogenetic clustering or overdispersion is evident across all taxonomic groups. Furthermore, sliding-window analyses indicate a trend towards greater phylogenetic clustering at coarse spatial scales, with slightly fewer clustered regions as analytical scale decreases (Appendix S3: Figs S4-S9). However, when slidingwindow results are restricted to statistically significant phylogenetic structure, the predominant pattern is non-significant community phylogenetic structure (at an $\alpha=0.05$ cutoff) across most assemblages for both NTI and NRI. This may be indicative of true neutrality in composition at these scales, or it may simply reflect the fact that broad-scale assemblages capture multiple communities composed by opposing forces (i.e. allopatry and sympatry), which suffer from loss of power as the analytical scale is decreased.

\section{Contemporary climate and arid-zone community structure}

At the IBRA (regional) scale of analysis, variables encompassing aspects of biogeographical history (e.g. taxonomic 

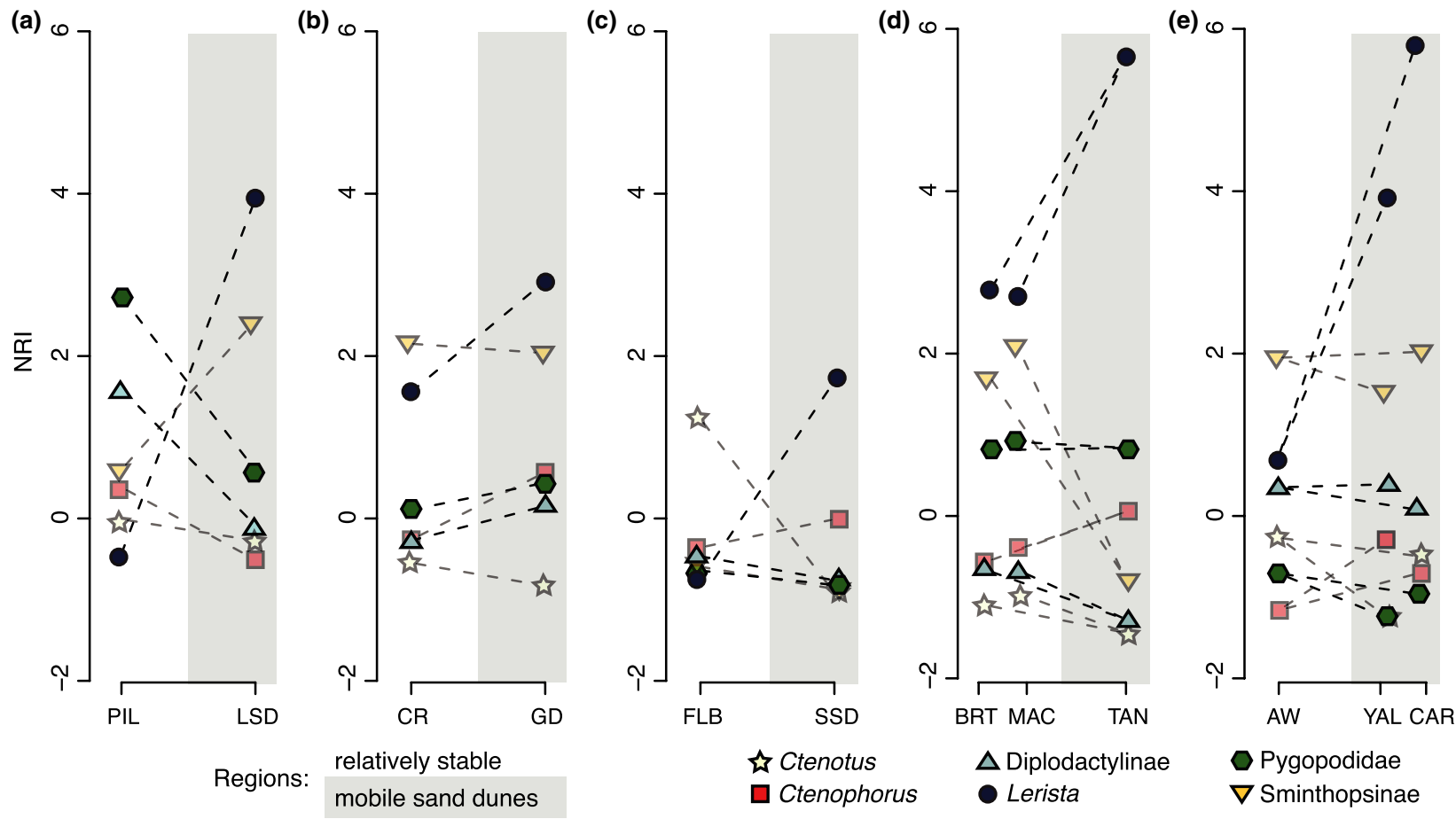

Figure 3 Changes in phylogenetic structure (based on net relatedness index, NRI) between relatively stable regions (left side of plot) and geographically proximate regions with mobile sand dunes indicate different responses among the six focal taxonomic groups to habitat stability across the Australian arid zone. Region codes and taxonomic group colours follow those in Fig. 1: (a) Pilbara versus the Little Sandy Desert, (b) Central Ranges versus the Gibson Desert, (c) Flinders Lofty Block versus the Simpson Strzelecki Dunefields, (d) the Burt Plain and the MacDonnell Ranges versus the Tanami, (e) the Avon Wheatbelt versus the Yalgoo and Carnarvon.

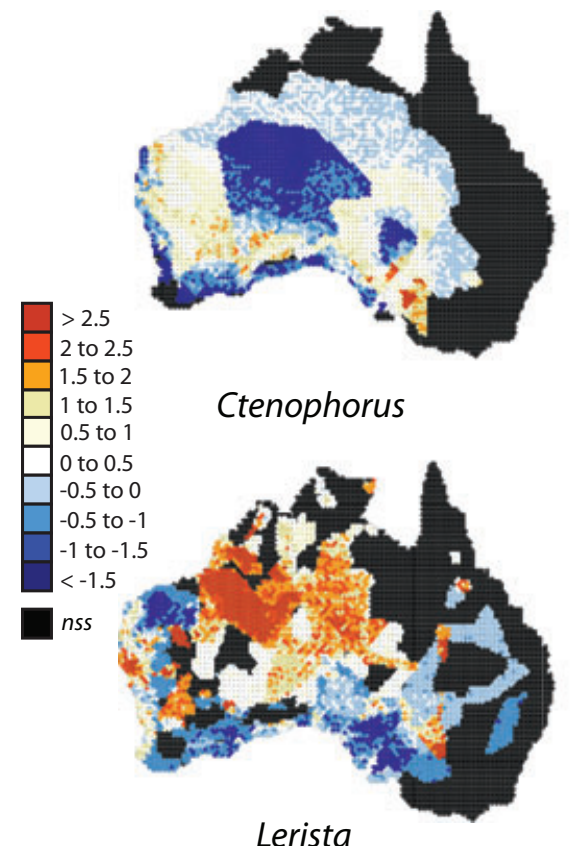

Lerista

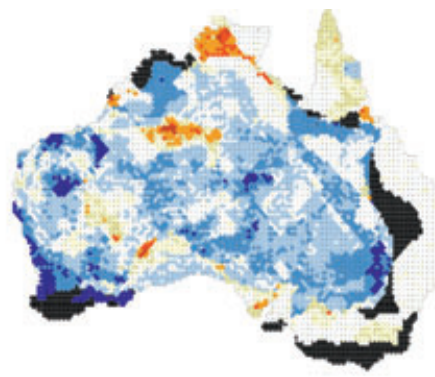

Ctenotus

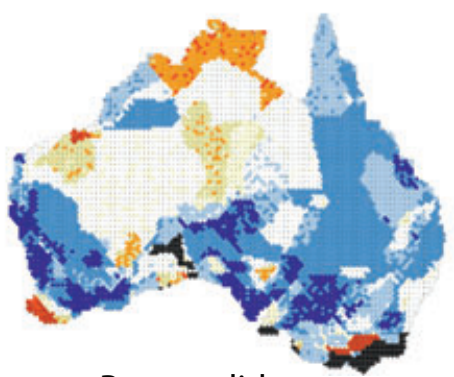

Pygopodidae

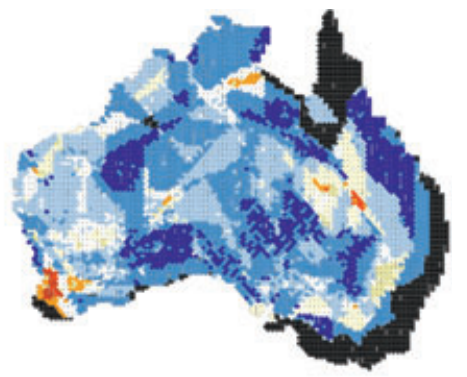

Diplodactylinae

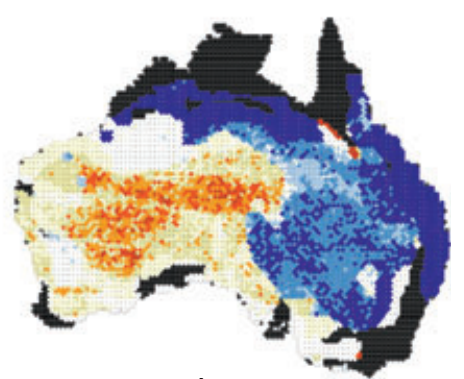

Sminthopsinae

Figure 4 Geographical distribution of phylogenetic community structure of the six focal taxonomic groups across the Australian arid zone, based on net relatedness index (NRI) calculated for assemblages sampled at the $50-\mathrm{km}$ scale. Assemblages with insufficient sample size (nss) for statistical analysis are shown in black. 


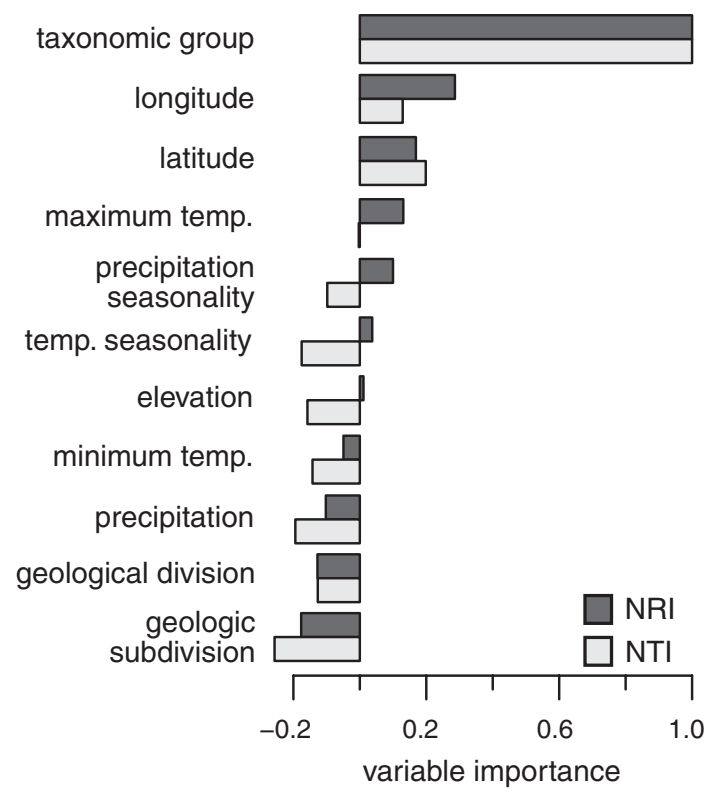

Figure 5 Variable importance as the percentage increase in mean square error (MSE), scaled against the most important variable (taxonomic group) from RANDOMForEsT models combining all six focal taxonomic groups (Ctenophorus, Ctenotus, Diplodactylinae, Lerista, Pygopodidae and Smithopsinae) for a particular index (net relatedness index, NRI, or nearest taxon index, NTI) across the Australian arid zone. Negative values indicate that the inclusion of a particular explanatory variable did not improve the model.

group, latitude and longitude) are consistently more important than proximate climatic factors in explaining community phylogenetic structure, as measured by either NRI or NTI (Fig. 5). The lack of consensus in community phylogenetic structure across taxonomic groups is consistent with previous observations that differential phylogenetic structure characterizes codistributed taxonomic groups (Bryant et al., 2008). However, we note that when each taxonomic group is analysed separately in the RANDOMFOREST framework, the importance of given biogeographical and climatic variables becomes apparent, with a larger percentage of the variance in phylogenetic structure (in NRI or NTI) explained, and with latitude or longitude always being the most important variable that explains the variance in NRI and NTI. For example, the models explain up to $63.3 \%$ of the variation in Sminthopsinae NRI, but account essentially for none of the variation in NRI for Ctenotus or Diplodactylinae (Appendix S3: Figs S10-S11).

Although climatic variables explain a much smaller proportion of the variance in phylogenetic structure, differential responses of taxonomic groups to certain variables may provide some explanation as to why broad-scale patterns differ between groups. In particular, maximum temperature was consistently one of the most important climatic variables for explaining variance in NRI and NTI within and between taxonomic groups, although the relationship between maximum temperature and community phylogenetic structure is not
Table 3 Mantel's $r$ coefficients for the relationship between phylogenetic beta diversity and explanatory factors within the six focal taxonomic groups, when all other factors, community beta diversity and geographical distance are held constant in a partial Mantel framework. Values significant at the $\alpha=0.05$ level are shown in bold.

\begin{tabular}{lccr}
\hline & Climate & Elevation & Centre \\
\hline Ctenophorus & $-\mathbf{0 . 1 5}$ & $-\mathbf{0 . 1 6}$ & -0.06 \\
Ctenotus & $-\mathbf{0 . 1 7}$ & $-\mathbf{0 . 1 9}$ & 0.07 \\
Diplodactylinae, centre 1 & $\mathbf{0 . 4 5 8}$ & 0.02 & $\mathbf{0 . 2 5}$ \\
Diplodactylinae, centre 2 & $-\mathbf{0 . 3 4 1}$ & 0.02 & $-\mathbf{0 . 2 6}$ \\
Lerista & -0.06 & -0.08 & $\mathbf{0 . 1 4}$ \\
Pygopodidae & 0.08 & -0.12 & 0.04 \\
Sminthopsinae & -0.01 & -0.05 & 0.08 \\
\hline
\end{tabular}

uniform. For example, partial dependence plots (Appendix S3: Fig. S12) of marginal change in NRI (or NTI) demonstrate a positive relationship between maximum temperature in the warmest quarter and phylogenetic clustering in Lerista, Ctenotus, Pygopodidae and Diplodactylinae, whereas Sminthopsinae and Ctenophorus show an inverse relationship between maximum temperature and clustering (i.e. greater phylogenetic clustering at lower maximum temperatures).

Regional similarity in phylogenetic beta diversity (PBD) is consistently and highly correlated with community beta diversity (CBD) for all taxonomic groups, as analysed with partial Mantel tests. Similar to the RANDOMForest results, the most important factors for explaining similarities in PBD differ across taxa. For example, climatic similarity between regions is an important determinant of $\mathrm{PBD}$ in three of the groups, and elevation and the centre of origin are significant for two groups (Table 3). Community structure (CBD) within each of the Pygopodidae and the Sminthopsinae is significantly related to $\mathrm{PBD}$, but not to any other explanatory variable.

\section{DISCUSSION}

While all six taxonomic groups in this study are arid-zone specialists, possessing a high degree of similarity in ecology and alpha diversity (Fig. 1), the underlying community phylogenetic structure was generally very different between groups. Not only did the magnitude of community phylogenetic patterns differ, but the directionality (phylogenetic clustering or overdispersion) of phylogenetic structure varied across these six lineages. This suggests that community phylogenetic structure in these taxonomic groups is the result of different underlying historical, ecological and evolutionary drivers (e.g. Fig. 3, Table 3), and that diversification in some groups may have acted to influence or limit the community structure in others.

Given the suggestion of common phylogenetic structure at the local scale within many analyses (Webb et al., 2008; Vamosi et al., 2009; Kooyman et al., 2011), the widespread lack of concordance observed here is somewhat surprising 
and raises the question of why codistributed taxonomic groups that are all arid specialists differ in the processes structuring their communities. The discordance across taxonomic groups may reflect differing centres of lineage persistence during Pleistocene aridity cycles, a supposition that would be generally supported by the presence of multiple localized refugial locations suggested by phylogeographical studies (see review in Byrne, 2008). Unlike Northern Hemisphere environments, where drastic shifts in climate resulted in fairly consistent refugial localities across codistributed species (Hewitt, 2000), Australian arid-zone fauna are thought to have withstood cyclic Pleistocene aridity in refugial locations at the margins of the arid zone or in localized pockets in situ (Byrne, 2008; Pepper et al., 2011). If regional patterns of phylogenetic clustering can be interpreted as evidence of in situ diversification, then these results (Figs 2 \& 3) may support a pattern of localized persistence specific to each taxonomic group. Consequently, although some habitat characteristics (e.g. elevation) are thought to contribute in some groups (e.g. diplodactyline geckos; Pepper et al., 2011), only one of the putative refugia (the Pilbara) showed greater phylogenetic clustering. Other localized patterns may also be related to habitat specialization. Lerista and Ctenophorus (excepting the Pilbara/Little Sandy Desert pairing in Fig. 3a) are always more clustered in geologically unstable regions than in areas with stable habitats. This could be due to the role of aridification in driving ecological speciation and diversification (Melville et al., 2001; Harmon et al., 2003; Skinner \& Lee, 2009) and these lizards' predominant use of sand habitats (Wilson \& Swan, 2008).

Perhaps the most surprising result was the commonly observed phylogenetic pattern of overdispersion across the arid zone, although the location and scale differed across taxa (Fig. 2). While it seems unlikely that large-scale biogeographical patterns would be (or could be) driven by competitive interactions (because competitive interactions are necessarily limited by an organism's ability to interact physically; Cavender-Bares et al., 2009; Wiens, 2011), the alternative explanation for this pattern - the persistent signal of widespread allopatric speciation (Webb et al., 2008) - may be even less likely across analytical scales. If the lack of concordance between taxonomic groups is driven by differing responses to aridity cycling during the Pleistocene, a scenario where clades within a taxonomic group are able to repeatedly come into contact and competitively exclude one another might be the most plausible of the two interpretations, suggesting an interaction between ecology and diversification history. This may be the driver of multiregion overdispersion in Sminthopsinae, one of the most mobile taxonomic assemblages included in these analyses (Haythornthwaite \& Dickman, 2006), and a group that shows no strong correlation between phylogenetic beta diversity and climate, elevation or centre of origin (Table 3).

While the regional results differed little with respect to sampling unit (i.e. $50-\mathrm{km}$ communities generally exhibited the same structure as their IBRA counterparts), our sliding- window results also supported a suite of previous observations that phylogenetic clustering is more common at coarse spatial scales (e.g. Swenson et al., 2006; Cavender-Bares et al., 2009). This phenomenon is thought to reflect the fact that larger regions can encompass greater environmental heterogeneity, which may limit closely related species in similar ways (Cavender-Bares et al., 2006; Swenson et al., 2006), as well as capturing a greater number of otherwise allopatric distributions, fragmented habitats and long-term dispersal limitation. As all of the communities in our analyses were sampled from regions that are larger than the scale at which strong competition will occur (the Darwin-Hutchinson zone sensu Vamosi et al., 2009), the predominance of phylogenetic clustering is also expected from an ecological perspective. Previous researchers have suggested that community structuring within Ctenotus is largely neutral, and the sheer size of the arid zone is responsible for the diversity of species within this genus (James \& Shine, 2000; Powney et al., 2010). While broad-scale analyses of Ctenotus assemblages (IBRA, $1000-\mathrm{km}$ and $500-\mathrm{km}$ analyses) indicated phylogenetic neutrality based on NRI, community similarity was significantly related to similarity in climate, elevation and in the distance to the centre of origin of the genus. Phylogenetic neutrality within assemblages of this group may be reflective of a variety of forces acting within Ctenotus, such as nichespace partitioning by activity periods (Pianka, 1969; Gordon et al., 2010) or the scale of analysis (finer-scale community analysis have indicated evenness in regions of the arid zone; Rabosky et al., 2011).

Many community phylogenetic studies have found that structuring mechanisms shift along gradients of elevation and precipitation (Cavender-Bares et al., 2006; Bryant et al., 2008; Graham et al., 2009). Steep environmental gradients can limit community membership to closely related species that possess key environmental tolerance traits (Graham et al., 2009). Topologically complex regions (e.g. high-elevation portions of the arid zone) are also thought to provide important mesic refugia during cycles of extreme aridity over geological timescales, acting to enhance the potential for microallopatric speciation or decrease the probability of lineage extinction (Pepper et al., 2011). While elevation alone did not account for a large proportion of variance in the models, partial dependence plots support this theory (see Appendix S3: Figs S12-S13), with a positive relationship between higher elevations and greater phylogenetic clustering as expected in the two Gondwanan-origin groups - Diplodactylinae and Pygopodidae - but also in Sminthopsinae. Even more important than elevation was maximum temperature in the warmest quarter, which was more highly ranked for explaining variance in NRI and NTI in four of the six groups.

\section{CONCLUSIONS}

This project represents one of the first attempts to quantify and contrast the community phylogenetic structure of multiple co-occurring assemblages at a regional scale. In the focal 
assemblages we examined, the greatest explanatory factors for phylogenetic structure are not primarily the result of current ecological forces; instead, phylogenetic structure appears to be fairly taxon- and region-specific. Climatic variables, such as maximum local temperature, account for far less variance than variables capturing biogeographical history (such as geographical location). Explicit tests of biogeographical scenarios that take species traits, environmental variables and phylogenetic histories into consideration may be useful to further clarify these findings (Leibold et al., 2010); it is, however, important that these tests be conducted within each group separately in addition to examining broad-scale patterns across all taxa.

Community phylogenetic analyses have a great potential to identify and elucidate the influences of biogeographical history and contemporary ecology in structuring biological communities, as well as generating interesting, testable hypotheses. Several important predictions would be interesting to test in the Australian arid zone across these taxa. For example, phylogenetically clustered assemblages of focal taxa in the central arid zone should be more functionally diverse (Prinzing et al., 2008) than codistributed phylogenetically even assemblages, such as Ctenophorus. For seemingly neutral communities, biological surveys and population genetic assessments would also be useful to examine whether low population density/abundance is correlated with neutral structure. More generally, community phylogenetic examinations of this type can reveal interesting regions, patterns and taxonomic groups to contrast in future analyses.

Areas of high diversity, by their very nature, are attractive and yet challenging targets for elucidating the factors structuring biodiversity, and many recent studies have made important inferences concerning the roles of biotic interactions and ecology by combining phylogenetic, distributional and traitbased information (see review in Vamosi et al., 2009). With the current crisis of species and habitat loss, this information becomes critical to preserving diversity (Moritz, 2002; Wiens \& Graham, 2005; Hendry et al., 2010). However, the logistics of tackling such questions are daunting when the answers inherently cut across disciplines. Community structure reflects (to varying degrees) both ecological and evolutionary processes, and comparing pattern and process between taxonomic groups can provide useful biological insights. Although ecological and biogeographical forces have resulted in broadly similar patterns across analytical scales within the taxonomic assemblages we examined, these indices did not generalize well between clades. Based on our work, we would caution against generalizing between even seemingly similar organisms, because similar groups may be responding differently to underlying biogeographical and ecological forces.

\section{ACKNOWLEDGEMENTS}

We thank D. Alvarado-Serrano, E.M. Humphries, M. Sistrom, the Knowles lab and two anonymous referees for helpful comments and insightful discussion, and T.K. Gabelman for assistance with ARcGIS. This research was supported by National Science Foundation grant DEB-0715487 to L.L.K.

\section{REFERENCES}

Bryant, J.A., Lamanna, C., Morlon, H., Kerkhoff, A.J., Enquist, B.J. \& Green, J.L. (2008) Microbes on mountainsides: contrasting elevational patterns of bacterial and plant diversity. Proceedings of the National Academy of Sciences USA, 105, 11505-11511.

Byrne, M. (2008) Evidence for multiple refugia at different time scales during Pleistocene climatic oscillations in southern Australia inferred from phylogeography. Quaternary Science Reviews, 27, 2576-2585.

Byrne, M., Yeates, D.K., Joseph, L., Kearney, M., Bowler, J., Williams, M.A.J., Cooper, S., Donnellan, S.C., Keogh, J.S., Leys, R., Melville, J., Murphy, D.J., Porch, N. \& Wyrwoll, K.-H. (2008) Birth of a biome: insights into the assembly and maintenance of the Australian arid zone biota. Molecular Ecology, 17, 4398-4417.

Cardillo, M. (2011) Phylogenetic structure of mammal assemblages at large geographical scales: linking phylogenetic community ecology with macroecology. Philosophical Transactions of the Royal Society B: Biological Sciences, 366, 2545-2553.

Cavender-Bares, J., Keen, A. \& Miles, B. (2006) Phylogenetic structure of Floridian plant communities depends on taxonomic and spatial scale. Ecology, 87, S109-S122.

Cavender-Bares, J., Kozak, K.H., Fine, P.V.A. \& Kembel, S.W. (2009) The merging of community ecology and phylogenetic biology. Ecology Letters, 12, 693-715.

Cutler, D.R., Edwards, T.C., Beard, K.H., Cutler, A., Hess, K.T., Gibson, J. \& Lawler, J.J. (2007) Random forests for classification in ecology. Ecology, 88, 2783-2792.

Dickman, C.R., Haythornthwaite, A.S., McNaught, G.H., Mahon, P.S., Tamayo, B. \& Letnic, M. (2001) Population dynamics of three species of dasyurid marsupials in arid central Australia: a 10-year study. Wildlife Research, 28, 493-506.

Doughty, P. \& Hutchinson, M.N. (2008) A new species of Lucasium (Squamata: Diplodactylidae) from the southern deserts of Western Australia and South Australia. Records of the Western Australian Museum, 25, 95-106.

Doughty, P., Oliver, P. \& Adams, M. (2008) Systematics of stone geckos in the genus Diplodactylus (Reptilia: Diplodactylidae) from northwestern Australia, with a description of a new species from the Northwest Cape, Western Australia. Records of the Western Australian Museum, 24, 247-265.

Drummond, A.J. \& Rambaut, A. (2007) BEAST: Bayesian evolutionary analysis by sampling trees. BMC Evolutionary Biology, 7, 214.

Fujioka, T., Chappell, J., Fifield, L.K. \& Rhodes, E.J. (2009) Australian desert dune fields initiated with Pliocene-Pleistocene global climatic shift. Geology, 37, 51-54. 
Gordon, C.E., Dickman, C.R. \& Thompson, M.B. (2010) Partitioning of temporal activity among desert lizards in relation to prey availability and temperature. Australian Ecology, 35, 41-52.

Graham, C.H. \& Fine, P.V.A. (2008) Phylogenetic beta diversity: linking ecological and evolutionary processes across space in time. Ecology Letters, 11, 1265-1277.

Graham, C.H., Parra, J.L., Rahbek, C. \& McGuire, J.A. (2009) Phylogenetic structure in tropical hummingbird communities. Proceedings of the National Academy of Sciences USA, 106, 19673-19678.

Harmon, L.J., Schulte, J.A., Larson, A. \& Losos, J.B. (2003) Tempo and mode of evolutionary radiation in iguanian lizards. Science, 301, 961-964.

Haythornthwaite, A.S. \& Dickman, C.R. (2006) Long-distance movements by a small carnivorous marsupial: how Sminthopsis youngsoni (Marsupialia: Dasyuridae) uses habitat in an Australian sandridge desert. Journal of Zoology, 270, 543-549.

Hendry, A.P., Lohmann, L.G., Conti, E., Cracraft, J., Crandall, K.A., Faith, D.P., Häuser, C., Joly, C.A., Kogure, K., Larigauderie, A., Magallón, S., Moritz, C., Tillier, S., Zardoya, R., Prieur-Richard, A.-H., Walther, B.A., Yahara, T. \& Donoghue, M.J. (2010) Evolutionary biology in biodiversity science, conservation, and policy: a call to action. Evolution, 64, 1517-1528.

Hewitt, G. (2000) The genetic legacy of the Quaternary ice ages. Nature, 405, 907-913.

Hijmans, R.J., Cameron, S.E., Parra, J.L., Jones, P.G. \& Jarvis, A. (2005) Very high resolution interpolated climate surfaces for global land areas. International Journal of Climatology, 25, 1965-1978.

Hutchinson, M.N., Doughty, P. \& Oliver, P.M. (2009) Taxonomic revision of the stone geckos (Squamata: Diplodactylidae: Diplodactylus) of southern Australia. Zootaxa, 2167, 25-46.

James, C.D. \& Shine, R. (2000) Why are there so many coexisting species of lizards in Australian deserts? Oecologia, 125, 127-141.

Jennings, W.B., Pianka, E.R. \& Donnellan, S. (2003) Systematics of the lizard family Pygopodidae with implications for the diversification of Australian temperate biotas. Systematic Biology, 52, 757-780.

Kembel, S.W. (2009) Disentangling niche and neutral influences on community assembly: assessing the performance of community phylogenetic structure tests. Ecology Letters, 12, 949-960.

Kembel, S.W., Cowan, P.D., Helmus, M.R., Cornwell, W.K., Morlon, H., Ackerly, D.D., Blomberg, S.P. \& Webb, C.O. (2010) Picante: $\mathrm{R}$ tools for integrating phylogenies and ecology. Bioinformatics, 26, 1463-1464.

Kooyman, R., Rossetto, M., Cornwell, W. \& Westoby, M. (2011) Phylogenetic tests of community assembly across regional to continental scales in tropical and subtropical rain forests. Global Ecology and Biogeography, 20, 707-716.
Leibold, M.A., Economo, E.P. \& Peres-Neto, P. (2010) Metacommunity phylogenetics: separating the roles of environmental filters and historical biogeography. Ecology Letters, 13, 1290-1299.

Macphail, M.K. (1997) Late Neogene climates in Australia: fossil pollen- and spore-based estimates in retrospect and prospect. Australian Journal of Botany, 45, 425-464.

McGill, B.J. (2010) Matters of scale. Science, 328, 575-576.

Melville, J., Schulte, J.A. \& Larson, A. (2001) A molecular phylogenetic study of ecological diversification in the Australian lizard genus Ctenophorus. Journal of Experimental Zoology, 291, 339-353.

Menkhorst, P. \& Knight, F. (2004) A field guide to the mammals of Australia, 2nd edn. Oxford University Press, Oxford.

Moritz, C. (2002) Strategies to protect biological diversity and the evolutionary processes that sustain it. Systematic Biology, 51, 238-254.

Morton, S.R., Stafford Smith, D.M., Dickman, C.R., Dunkerley, D.L., Friedel, M.H., McAllister, R.R.J., Reid, J.R.W., Roshier, D.A., Smith, M.A., Walsh, F.J., Wardle, G.M., Watson, I.W. \& Westoby, M. (2011) A fresh framework for the ecology of arid Australia. Journal of Arid Environments, 75, 313-329.

Nylander, J.A.A. (2004) MrModeltest v2. Program distributed by the author. Evolutionary Biology Centre, Uppsala University.

Pepper, M., Fujita, M.K., Moritz, C. \& Keogh, J.S. (2011) Palaeoclimate change drove diversification among isolated mountain refugia in the Australian arid zone. Molecular Ecology, 20, 1529-1545.

Pianka, E.R. (1969) Sympatry of desert lizards (Ctenotus) in Western Australia. Ecology, 50, 1012-1030.

Pianka, E.R. (1972) Zoogeography and speciation of Australian desert lizards: an ecological perspective. Copeia, 1972, 127-145.

Powney, G.D., Grenyer, R., Orme, C.D.L., Owens, I.P.F. \& Meiri, S. (2010) Hot, dry and different: Australian lizard richness is unlike that of mammals, amphibians and birds. Global Ecology and Biogeography, 19, 386-396.

Prinzing, A., Reiffers, R., Braakhekke, W.G., Hennekens, S.M., Tackenberg, O., Ozinga, W.A., Schaminée, J.H.J. \& van Groenendael, J.M. (2008) Less lineages - more trait variation: phylogenetically clustered plant communities are functionally more diverse. Ecology Letters, 11, 809-819.

Rabosky, D.L., Reid, J., Cowan, M.A. \& Foulkes, J. (2007) Overdispersion of body size in Australian desert lizard communities at local scales only: no evidence for the Narcissus effect. Oecologia, 154, 561-570.

Rabosky, D.L., Cowan, M.A., Talaba, A.L. \& Lovette, I.J. (2011) Species interactions mediate phylogenetic community structure in a hyperdiverse lizard assemblage from arid Australia. The American Naturalist, 178, 579-595.

Shoo, L.P., Rose, R., Doughty, P., Austin, J.J. \& Melville, J. (2008) Diversification patterns of pebble-mimic dragons are consistent with historical disruption of important habi- 
tat corridors in arid Australia. Molecular Phylogenetics and Evolution, 48, 528-542.

Skinner, A. \& Lee, M.S.Y. (2009) Body-form evolution in the scincid lizard clade Lerista and the mode of macroevolutionary transitions. Evolutionary Biology, 36, 292-300.

Smith, L.A. \& Adams, M. (2007) Revision of the Lerista muelleri species-group (Lacertilia: Scincidae) in Western Australia, with a redescription of L. muelleri (Fischer, 1881 ) and the description of nine new species. Records of the Western Australian Museum, 23, 309-357.

Swenson, N.G. (2009) Phylogenetic resolution and quantifying the phylogenetic diversity and dispersion of communities. PLoS ONE, 4, e4390.

Swenson, N.G., Enquist, B.J., Pither, J., Thompson, J. \& Zimmerman, J.K. (2006) The problem and promise of scale dependency in community phylogenetics. Ecology, 87, 2418-2424.

Vamosi, S.M., Heard, S.B., Vamosi, J.C. \& Webb, C.O. (2009) Emerging patterns in the comparative analysis of phylogenetic community structure. Molecular Ecology, 18, 572-592.

Webb, C.O., Ackerly, D.D., McPeek, M.A. \& Donoghue, M.J. (2002) Phylogenies and community ecology. Annual Review of Ecology and Systematics, 33, 475-505.

Webb, C.O., Cannon, C.H. \& Davies, S.J. (2008) Ecological organization, biogeography, and the phylogenetic structure of rainforest tree communities. Tropical forest community ecology (ed. by W. Carson and S. Schnitzer), pp. 79-97. Blackwell, Oxford.

Wiens, J.J. (2011) The niche, biogeography and species interactions. Philosophical Transactions of the Royal Society B: Biological Sciences, 366, 2336-2350.

Wiens, J.J. \& Graham, C.H. (2005) Niche conservatism: integrating evolution, ecology, and conservation biology. Annual Reviews of Ecology, Evolution, and Systematics, 36, 519-539.
Wilson, S. \& Swan, G. (2008) A complete guide to reptiles of Australia, 2nd edn. New Holland, Sydney.

\section{SUPPORTING INFORMATION}

Additional Supporting Information may be found in the online version of this article:

Appendix S1 GenBank numbers, BEAsT models and loci. Appendix S2 Community phylogenetic structure with species-pool null model.

Appendix S3 Supplementary figures.

\section{BIOSKETCHES}

Hayley C. Lanier is a postdoctoral fellow at the University of Michigan whose research focuses on the systematics, phylogeography and population genetics of small mammals. She also studies issues related to the statistical resolution of species trees, and the uses of phylogenetic techniques for comparative analyses of evolutionary histories.

Danielle L. Edwards is interesting in disentangling the effects of life history, physiology, phenotype and ecology on population history in a comparative context. She is currently a postdoctoral research associate in the lab of Gisella Caccone at Yale University.

L. Lacey Knowles is a professor whose research focuses on the demographic and selective processes driving species divergence. Her research also addresses the methodological and statistical issues involved in studying species diversification, phylogeography and phylogenetic relationships of recently diverged and rapidly speciating taxa.

Editor: Brett Riddle 